\title{
Research Article \\ Effect of a Balanced Concentration of Hydrogen on Graphene CVD Growth
}

\author{
S. Chaitoglou, E. Pascual, E. Bertran, and J. L. Andujar \\ FEMAN Group, IN2UB, Departament de Física Aplicada i Òptica, Universitat de Barcelona, \\ Martí i Franquès 1, 08028 Barcelona, Catalonia, Spain
}

Correspondence should be addressed to S. Chaitoglou; schaitog@gmail.com

Received 6 October 2015; Revised 23 December 2015; Accepted 30 December 2015

Academic Editor: Ungyu Paik

Copyright (C) 2016 S. Chaitoglou et al. This is an open access article distributed under the Creative Commons Attribution License, which permits unrestricted use, distribution, and reproduction in any medium, provided the original work is properly cited.

The extraordinary properties of graphene make it one of the most interesting materials for future applications. Chemical vapor deposition (CVD) is the synthetic method that permits obtaining large areas of monolayer graphene. To achieve this, it is important to find the appropriate conditions for each experimental system. In our CVD reactor working at low pressure, important factors appear to be the pretreatment of the copper substrate, considering both its cleaning and its annealing before the growing process. The carbon precursor/hydrogen flow ratio and its modification during the growth are significant in order to obtain large area graphene crystals with few defects. In this work, we have focused on the study of the methane and the hydrogen flows to control the production of single layer graphene (SLG) and its growth time. In particular, we observe that hydrogen concentration increases during a usual growing process (keeping stable the methane/hydrogen flow ratio) resulting in etched domains. In order to balance this increase, a modification of the hydrogen flow results in the growth of smooth hexagonal SLG domains. This is a result of the etching effect that hydrogen performs on the growing graphene. It is essential, therefore, to study the moderated presence of hydrogen.

\section{Introduction}

This paper aims to explore additional routes for graphene growth by chemical vapor deposition (CVD) [1], which may be more suitable for the production of this emerging material.

Graphene consists of a single atomic layer of carbon with a honeycomb structure [2].

Graphene has attracted the attention because of its outstanding characteristics. High mechanical strength [3], carrier mobility [4], electrical conductivity [1], and transparency [5] are some of them, making it a promising material in applications concerning photovoltaics [6,7], transparent electrodes $[8,9]$, and gas sensors [10]. From all the established methods of graphene synthesis [11-13], CVD appears as the uppermost, as it enables the growth of larger domains of graphene. Usually, the growth of graphene directly takes place in preannealed copper foil. Copper foil is one of the preferred substrates for the CVD growth as it permits the production of large scale, single layer graphene domains [14]. Carbon atoms show a low solubility into the copper. More carbon species remain in the surface of the foil participating in the formation of the graphene sheet [15].

A conventional CVD growth method consists of a continuous flow of carbon gas precursor $/ \mathrm{H}_{2} / \mathrm{Ar}$ mixture in order to generate the graphene growth [16]. In the present work we have examined how the variation of the $\mathrm{CH}_{4} / \mathrm{H}_{2}$ ratio during the growth affects the etching mechanism that the hydrogen performs. We present the optimum results which allowed the growth of large, up to $30 \times 30 \mu \mathrm{m}^{2}$, graphene crystals with the lowest number of defects.

According to the previous results of Li et al. [17], we used methane as carbon precursor in all the growing processes. The steps for the graphene growth are the following:

(1) Exposure of $\mathrm{Cu}$ to methane, argon, and hydrogen.

(2) Catalytic decomposition of methane on $\mathrm{Cu}$ to mainly form $\mathrm{CH}_{y}$ species.

(3) Depending upon the temperature, methane pressure, methane, argon and hydrogen flow, and partial 


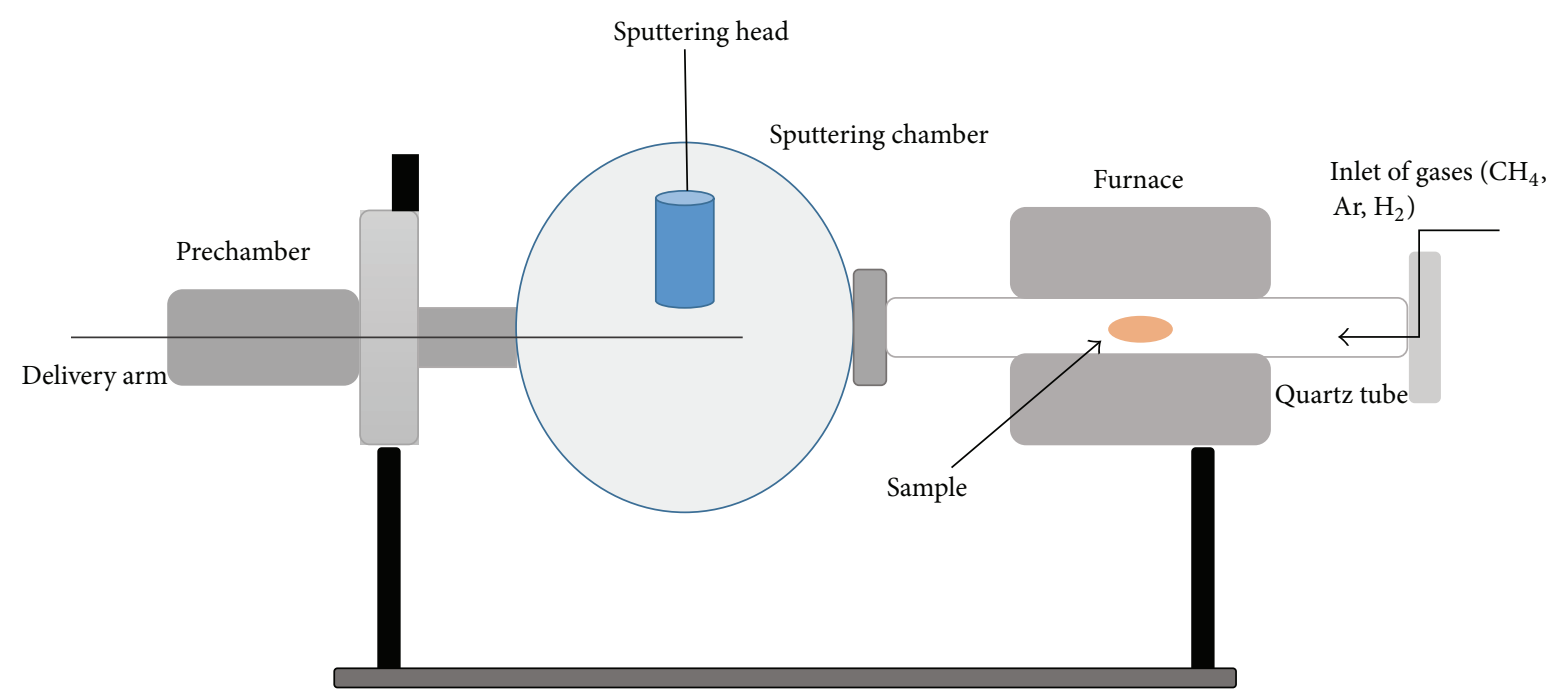

FIGURE 1: Schematic illustration of GRAPHman reactor for DC plasma and CVD process of the FEMAN group at University of Barcelona.

pressure, the $\mathrm{Cu}$ surface being obtained undersaturated, saturated, or supersaturated with $\mathrm{CH}_{y}$ species.

(4) Formation of nuclei as a result of local supersaturation of $\mathrm{CH}_{y}$.

(5) Nuclei grow to form graphene islands.

(6) Full $\mathrm{Cu}$ surface coverage by graphene under specific temperature flow rates and pressure conditions.

In this work we pursue to grow graphene without voids or defects forming large domains, by means of a modified method. Continuous graphene film without defects is desired in order for the material to maintain its exceptional properties and to be useful for applications.

\section{Experimental Part}

The whole process was carried out in a suitable CVD oven (schematically drawn in Figure 1). The reactor consists of a furnace, where the CVD process takes place, coupled to a spherical chamber with a sputtering system. This allows us to deposit different thin films (copper-nickel on silicon) in ultrapure conditions (without oxygen) and to perform directly the graphene growth without exposing the substrate to the ambient. By this method, all the treatments, since the cleaning process and annealing of the copper foil or the deposition of the metal catalyst by sputtering, can be performed "in situ" by a single run. For the evaluation of the obtained graphene we used Raman spectroscopy and mapping as well as scanning electron microscopy (SEM). The SEM images were taken with a JEOL JSM 7100F microscope in $5 \mathrm{keV}$. The Raman spectroscopy was performed with a Jobin-Yvon LabRam HR 800 system. A green laser with a $2 \mu \mathrm{m}$ spot diameter has been used. AFM image has been obtained with an AFM Multimode 8, electrònica Nanoscope $\mathrm{V}$ (Bruker), and operated by the NOVA software.
2.1. Pretreatment of Copper Foil. Polycrystalline copper foil $75 \mu \mathrm{m}$ thick and 99\% pure was cut in pieces of $2 \times 2 \mathrm{~cm}^{2}$. The foil piece is cut in pieces of $2 \times 2 \mathrm{~cm}^{2}$. It is precleaned with acetone and isopropanol immersed in an ultrasonic bath for 10 minutes. Then it is being introduced into the CVD reactor. In other works the application of argon/hydrogen of helium/hydrogen plasma for the effective pretreatment of the copper foil is being introduced. Plasma pretreatment is moreover found to improve the crystallinity of the formed graphene [18]. Here we present the performance of hydrogen plasma for the same purpose. From previous studies made in our group [19], we have been able to optimize the parameters necessary to use in order to reduce the native copper oxide layer. According to these results, first, a background pressure of $7 \times 10^{-4} \mathrm{~Pa}$ is applied with a turbomolecular pump to secure that the whole process is taking place under high purity conditions. Radio Frequency (RF) hydrogen plasma is then applied to chemically reduce the copper substrate. To generate the plasma we introduce hydrogen with $20 \mathrm{sccm}$ flow rate and applied $100 \mathrm{~W}$ RF power at $20 \mathrm{~Pa}$. The hydrogen radicals are are reacting with the copper oxide

$$
\mathrm{CuO}+2 \mathrm{H} \longrightarrow \mathrm{Cu}+\mathrm{H}_{2} \mathrm{O}
$$

2.2. CVD Synthesis. CVD synthesis is performed after the plasma etching and the reduction of copper foil inside a quartz tube surrounded by an oven. Figure 1 shows a photo and a scheme of the GRAPHman reactor where the whole process is conducted. Hydrogen plasma for copper reduction is excited by the cathode of the magnetron sputtering head located in the left part on top of the spherical chamber. On the right part, the quartz tube is inserted inside a tubular furnace that can reach temperatures up to $1040^{\circ} \mathrm{C}$.

All the steps of the process can be controlled by a computer program; moreover, in order to secure the high purity conditions under which the graphene growth is carried 
out, the reactor is pumped to high vacuum (down to $6 \times$ $10^{-4} \mathrm{~Pa}$ ) with a turbomolecular pump. In addition, the tubular furnace is heated to $1040^{\circ} \mathrm{C}$.

Various publications have reported the crucial impact of this temperature concerning the CVD synthesis of graphene on copper using methane as a carbon precursor [20]. In our case, after exploring a wide range of experimental conditions, we conclude that the best quality graphene is obtained at this temperature. Studies reveal the possibility of CVD graphene growth in lower temperatures. However, it requires working at higher pressures outside of the range that our system can reach [21]. The pressure was stabilized at $20 \mathrm{~Pa}$ by automatically controlling the position of a variable conductance valve. Flows of $20 \mathrm{sccm}$ of hydrogen and $5 \mathrm{sccm}$ of methane were introduced in the chamber and, in order to compare the characteristics of the obtained graphene crystals, we kept running the deposition process for $20 \mathrm{~min}$ (Sample A) and $40 \mathrm{~min}$ (Sample B) [14, 22]. After these running times, the flows of methane and hydrogen were stopped and the system was cooled down to room temperature in high vacuum $\left(10^{-4} \mathrm{Pascal}\right)$. This operation prevents the oxidation of the samples under atmosphere exposition [23].

\section{Results and Discussion}

3.1. Copper Oxide Reduction. During the hydrogen plasma treatment the hydrogen radicals react with the copper oxide reducing it to metallic copper [24]. We use optical emission spectroscopy to determine and evaluate the reduction of the copper surface. The outcoming light from the discharge was collected by a spectrophotometer (Stellarnet EPP2000C), which operated in the range $300-850 \mathrm{~nm}$. The evolution of the $\mathrm{OH}$ radical integral spectral intensity in the spectral range of $305-330 \mathrm{~nm}$ is a sufficient tool to evaluate the removal of the oxide [25]. Results show that the intensity of the peaks corresponding to $\mathrm{OH}$ radicals decrease after $5 \mathrm{~min}$ of plasma treatment. In particular, the first peak at $305 \mathrm{~nm}$ decreases from 22.6 to 5.9 a.u., as we can see in Figure 2. The reduction in the $\mathrm{OH}$ concentration reveals the reduction of the oxygen radicals (as a result of the oxide reduction). Nevertheless, as the copper oxide cannot be completely removed, the peak does not decrease more with further treatment [26]. Figure 3(a) shows the copper foil used as a substrate before (up and down sample) and after (medium sample) the hydrogen etching and the graphene growth. Graphene protects copper from oxidation which explains that even after the passage of days the reflected color of the substrate does not change [27]. Copper foil before etching appears with a higher pitch of red because of the copper oxide layer on the top. After the treatment it appears brighter and large crystalline domains can be distinguished at naked eyes. This process is much more time sufficient, compared to the usual preannealing required for the $\mathrm{Cu}$ substrates taking up to several hours [28].

As an additional tool to characterize the copper surface we used electron backscattered diffraction (EBSD). It is a large area imaging method which detects the different index copper facets. Here, EBSD is used in order to demonstrate

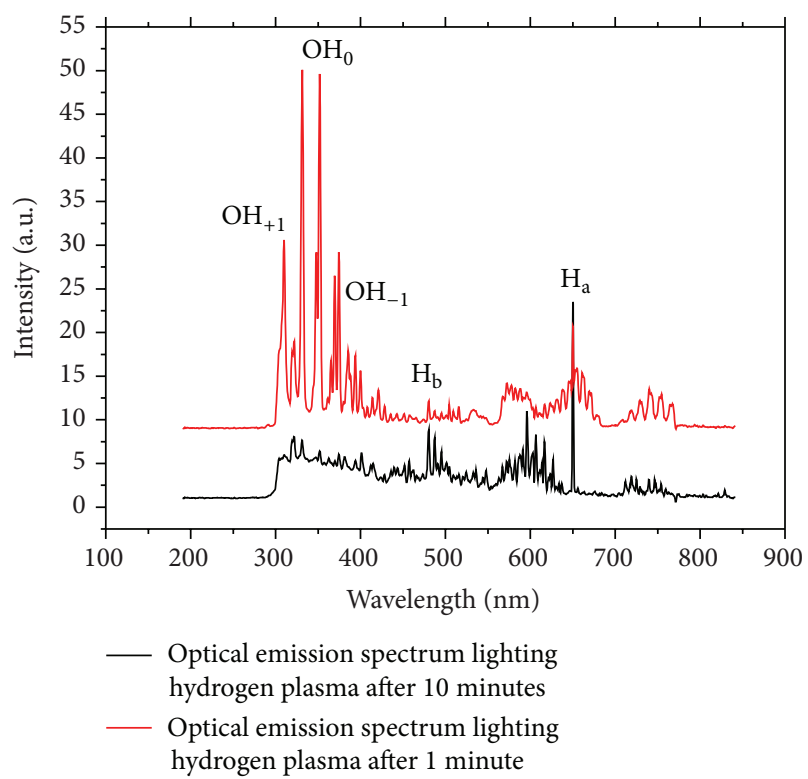

FIGURE 2: Optical emission spectroscopy of hydrogen plasma during reduction of copper: after 1 minute (red line) and after 5 minutes (black line). The reduction of the $\mathrm{OH}$ radical peak intensity in the spectral range of $305-330 \mathrm{~nm}$ reveals the removal of the oxide layer.

the growth of the copper grains after the $\mathrm{H}_{2}$ plasma and the growth process, with respect to the as-received copper foil. In Figure 3 we provide the EBSD mapping of untreated polycrystalline copper foil of $75 \mu \mathrm{m}$ (Figure 3(b)), copper foil after 20 minutes growth (Figure 3(c)), and copper foil after 40 minutes growth (Figure 3(d)). As we see, the grains of the untreated copper have various sizes with most of them not overcoming $\sim 30 \mu \mathrm{m}^{2}$. The boundaries between the grains are randomly oriented. During the heating and the growth the necessary energy is provided to the grains in order to increase in size. In our experiments the heating is taking place under no presence of gas, in very high vacuum, and in the order of $10^{-4} \mathrm{~Pa}$. The growth is taking place under the presence of hydrogen and methane, in a pressure of $\sim 20 \mathrm{~Pa}$ and in a temperature of $\sim 1040^{\circ} \mathrm{C}$. As we see in Figures 3(c) and 3(d), with the longer growth, the grains grow bigger. We see gains of sizes up to $\sim 2000 \mu \mathrm{m}^{2}$ for the longer growth. The edges between the grains now are much straighter. These factors affect the quality of the grown graphene later. Graphene needs smooth and large grains to grow over. Usually the boundaries are defect sites for the graphene, so the less possible boundaries mean the less possible defects.

3.2. Graphene Grown Domains. Scanning electron microscopy was used to evaluate the morphology of the obtained graphene. The micrographs of Figures 4(a), 4(b), and 4(c) were taken with a JEOL JSM 7100F microscope. The electron beam has energy of $5 \mathrm{keV}$. In sample A (Figure 4(a)), graphene crystals were obtained [29]. In the figure we see the formation of crystals with different morphologies. There are present crystals with a "butterfly" shape (blue circle) and others formed with four lobes and dendritic extensions (red circle). Shape and symmetry of graphene crystals depend on the 


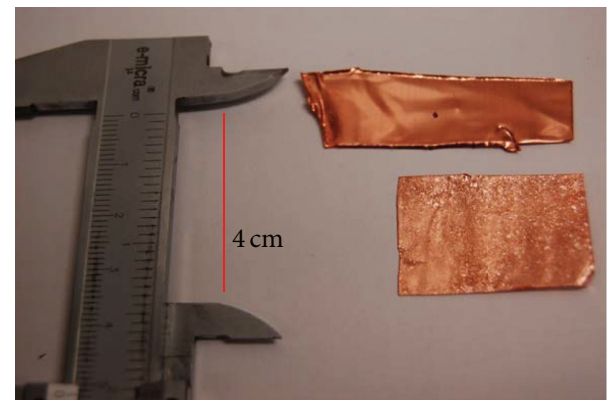

(a)

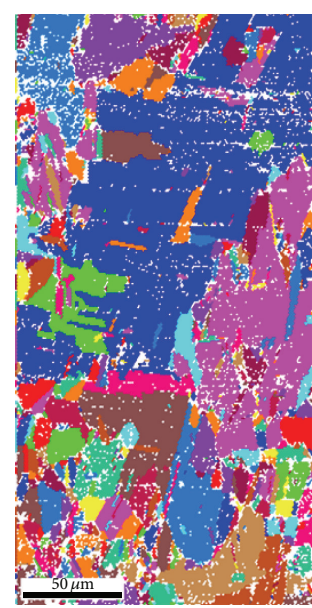

(b)

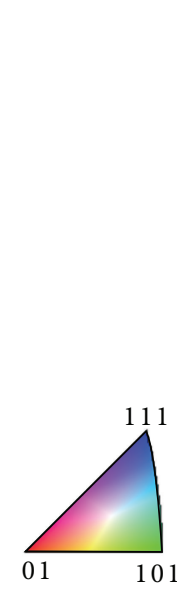

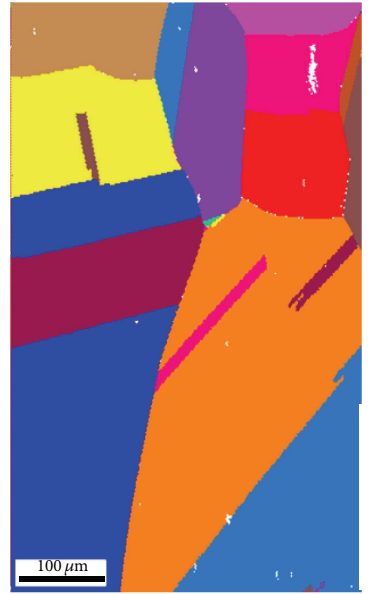

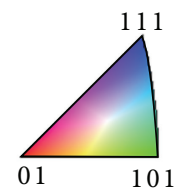

(c)

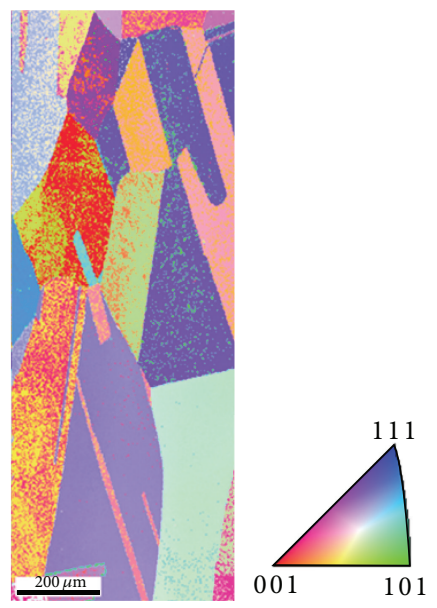

(d)

Figure 3: (a) Polycrystalline copper foil before (up piece) and after (down piece) the hydrogen etching. The difference in the surface color indicates the deoxidation of it. The red line indicates $4 \mathrm{~cm}$. EBSD maps of (b) the as-received polycrystalline copper foil (scale bar $50 \mu \mathrm{m}$ ), (c) the foil after plasma annealing and 20 minutes graphene growth at $1040^{\circ} \mathrm{C}$, (scale bar $100 \mu \mathrm{m}$ ), and (d) the foil after plasma annealing and 50 minutes graphene growth (scale bar $200 \mu \mathrm{m}$ ). The triangle palette matches the copper facets orientation with the relative color and is common for all three maps.

orientation of the copper crystalline domain of the substrate. According to the model of Meca et al., the four lobes with dendritic extension crystals are being grown on $\mathrm{Cu}$ (100) planes, whereas the crystals with the "butterfly-like" shape are being grown on $\mathrm{Cu}$ (221) planes [30]. The pictures were taken directly in the copper substrate without any transfer in advance. The lobes of the crystals are about $30 \mu \mathrm{m}$ (growth velocity $1.5 \mu \mathrm{m} / \mathrm{min}$ ). The growth ratio is independent of the crystal morphology. More favourable appears to be the growth of crystals with the four lobes with dendritic extensions morphology. The crystals present a medium nucleation density in most parts of the substrate as SEM pictures show in Figure 4(a). Large areas of the substrate are not covered by graphene. Therefore, longer growth time is necessary. We consider that a process provides a high-quality graphene when, on the substrate, only "monolayer graphene" grows.
In sample B (Figure 4(b)), with double growth process time and same deposition conditions (in particular, gas flows), we have observed that the size of the graphene flakes does not change appreciably, but the nucleation density increases significantly. Although now flakes do not appear with such regular shapes. This phenomenology seems to be a clear effect of the presence of hydrogen species bombarding the carbon adatoms with high mobility. Then, carbon adatoms would move along the hot surface and on the boundaries of the graphene islands. In this hypothesis, hydrogen bombardment would remove the carbon atoms which are not well positioned in the energetically favourable sites. Furthermore, this phenomenon seems to be associated with an effect of anisotropic etching of hydrogen along the crystal direction of polycrystalline copper substrate during the growth of graphene [31, 32] (Figure 4(b)). 


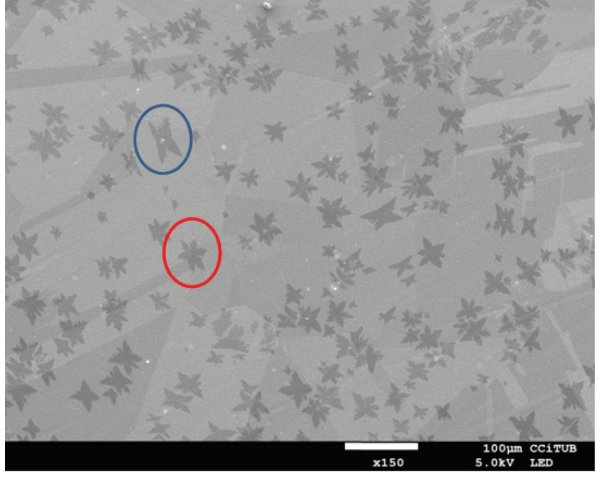

(a)

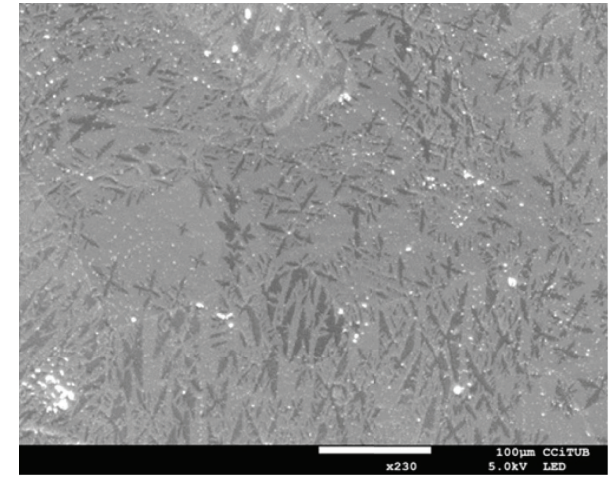

(b)

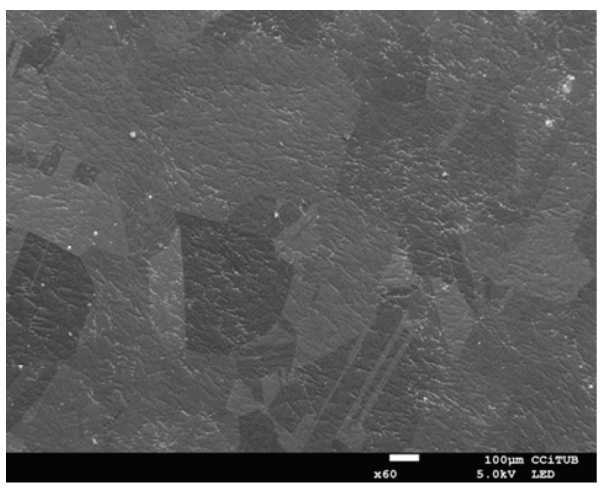

(c)

FIGURE 4: Scanning electron microscope pictures of graphene domains on copper foil after (a) $20^{\prime}$ growth. Four lobes with dendritic extension (blue circle) and butterfly-like (red circle) graphene islands are grown with a size of $40 \mu \mathrm{m}$. (b) 40 minutes growth. The nucleation density is higher but the domains are etched because of the high hydrogen concentration. (c) $40^{\prime}$ growth with reduced hydrogen flow. During the first $20^{\prime}$ of growth, the hydrogen flow was $20 \mathrm{sccm}$ and in the rest of the process $\mathrm{H}_{2}$ flow was stopped. This method avoids the etching observed in the previous sample. Most of the substrate appeared covered with graphene. The scale bar is always at $100 \mu \mathrm{m}$.

The white spots observed in the pictures of Figure 4 correspond to oxides formed on the surface [33]. Despite the plasma etching and the high prevacuum, in the order of $10^{-4} \mathrm{~Pa}$, to ensure the highest pure conditions, at high temperature (above $1000^{\circ} \mathrm{C}$ ) even the smallest amount of oxygen can be highly active. This may originate from impurities in the walls of the reactor and the inside of the oven. Even after a precise cleaning of the oven it was not possible to completely eliminate these oxides. Characterising the oxides by EDS analysis revealed that they are $\mathrm{SiO}_{x}$. This observation has been mentioned to the latest works as well [34]. These silicon particles are introduced to the copper foil from the quartz tube during the heating process. It has been studied in other works that they can function as nucleation center and catalyst for the graphene growth [43]. In our SEM images no matching between the nucleation center of each graphene crystal and the $\mathrm{SiO}_{2}$ particles appears; therefore, we do not consider that they affect the process.

Taking into account these observations, we performed a graphene growth (sample C, Figure 4(c)) of 40 minutes under the same pressure and growth rates, but pausing the hydrogen flow after the first 20 minutes had passed (Table 1). All the growths have been performed inside the same quartz tube. The methane flow remains open during the whole time (40 minutes). To conclude this growing process, in phases, we reduce the hydrogen concentration in the chamber during the last 20 minutes. Although the hydrogen is not completely evacuated from the chamber, some quantities always remain inside. This is a result of the inability of the turbomolecular pump to sufficiently evacuate low mass atoms as hydrogen. In the range of pressures that we have used, the hydrogen concentration increase had been balanced by the reduction of the hydrogen flow in order to keep constant the hydrogen concentration. So, the hydrogen amount that remains in the chamber is enough for performing smoothly the effects induced during the graphene growth. Hydrogen has a double role during the graphene growth. It activates the surface-bound carbon that leads to monolayer growth and function as an etching reagent that controls the size and morphology of the resulting graphene domains. A careful design should ensure that the flow and concentration of the gases is suitable for facilitating the growth of graphene. If the concentration of hydrogen is too high, as we see in Figure 4(b), the formed graphene can easily be taken away by the etching activity of hydrogen flow. The reduced hydrogen concentration of the sample $\mathrm{C}$ avoids this, permitting the 
TABLE 1: Experimental conditions of different samples of growth graphene. The variation in the precursor flows, growth time, pressure, and substrate pretreatment are being demonstrated.

\begin{tabular}{|c|c|c|c|c|c|c|c|}
\hline Sample & $\begin{array}{l}\text { Methane } \\
\text { flow (scc) }\end{array}$ & \multicolumn{2}{|c|}{ Hydrogen flow (sccm) } & $\begin{array}{l}\text { Total time } \\
\text { (min) }\end{array}$ & Pressure $(\mathrm{Pa})$ & Pretreatment & Crystal size $\left(\mu \mathrm{m}^{2}\right)$ \\
\hline $\bar{A}$ & 5 & \multicolumn{2}{|c|}{20} & 20 & 20 & Hydrogen plasma etching & $\sim 50$ \\
\hline B & 5 & \multicolumn{2}{|c|}{20} & 40 & 20 & Hydrogen plasma etching & $\sim 60$ \\
\hline $\mathrm{C}$ & 5 & $\begin{array}{l}\text { First } 20 \mathrm{~min} \text {, } \\
20 \mathrm{sccm}\end{array}$ & $\begin{array}{l}\text { Last } 20 \mathrm{~min} \text {, } \\
0 \mathrm{sccm}\end{array}$ & 40 & 20 & Hydrogen plasma etching & $\sim 60$ (full cover) \\
\hline $\mathrm{D}$ & 5 & \multicolumn{2}{|c|}{20} & 20 & 20 & Chemical etching & Bad shaped \\
\hline
\end{tabular}

total coverage of the surface by graphene [35]. SEM images show the complete coverage of the substrate with graphene (Figure 4(c)). Only in few parts, the grain boundaries are visible. The boundaries affect the quality of the film. In these parts we can distinguish that the graphene domains that form the continuous graphene film have the same average size as in samples A and B. This observation comes in contrast to previous ones which suggest that the absence of hydrogen during the growth step leads to smaller graphene domains [36]. We consider that in a stable total pressure the graphene domains size remains stable and that the hydrogen variation affects the etching mechanism and therefore the domains shape. In Figure 5 the flows of methane and hydrogen are presented as a function of the growth time for samples B and $\mathrm{C}$. The hydrogen flow is 0 for the second step of the growth in sample $\mathrm{C}$. This moderates the hydrogen etching effect, resulting in increase of the graphene surface coverage from $25 \%$ to $99 \%$ of the total surface. The graphene coverage was measured by Image J image processing program.

Raman spectroscopy results, performed with a JobinYvon LabRam HR 800 system, are shown in Figure 6. The Raman spectrum in Figure 6(a) shows $G$ and $2 \mathrm{D}$ peaks with an intensity $I_{2 \mathrm{D}} / I_{\mathrm{G}} \sim 1.5$ (red and green colored spectra, corresponding to graphene grown in the two different faces of copper foil). This confirms the high quality, monolayer thickness of graphene [37] grown in the above conditions.

Although, as the spectrum is taken directly from graphene deposited on the copper without any transfer process, some background noise in the signal appears because of photoluminescence from the copper substrate, a background subtraction during the data analysis was performed to evidence the signal coming from graphene [38]. To obtain an enhanced Raman spectrum from graphene, a common transferring process was followed [39]. A polymer support, poly(methyl methacrylate) (PMMA), was applied via spin-coating on top of the graphene/copper substrate. Then, it was immersed into $\mathrm{FeCl}_{3}$ to etch the copper and the graphene/PMMA was transferred onto a $\mathrm{SiO}_{2}$ (90 nm thick)/Si substrate (Figure 6(b)) and quartz substrate (Figure 6(a), black colored spectra). After transferring the graphene from the metal catalyst to $\mathrm{SiO}_{2}$ substrate the background noise disappears and the characteristic peaks appear sharper and better shaped. The characteristic $\mathrm{D}+\mathrm{D}^{\prime \prime}$ peak is also visible (Figure 6(b)) [40]. The 2D peak appears upshifted with respect to the spectra taken directly from the copper foil. The $I_{2 \mathrm{D}} / I_{\mathrm{G}}$ ratio now is 2.5. Similar upshifting and ratio increase appears in the spectra obtained from

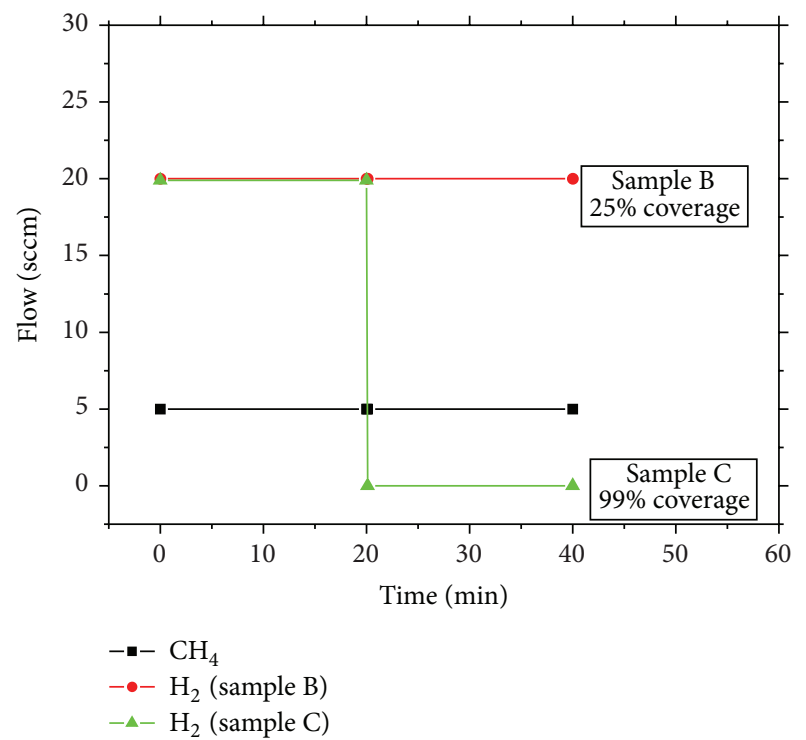

FIGURE 5: The flows of methane and hydrogen are demonstrated as a function of the growth time. Methane flow is stable. Hydrogen flow is stable for sample B. In sample C a 2-step recipe is followed. In the second step the hydrogen flow is stopped. This moderates the hydrogen etching effect, resulting in increase of the graphene surface coverage from $25 \%$ to $99 \%$ of the total surface.

the quartz substrate. Although a small D peak is visible, possible result of defects is introduced in the graphene sheet during the transfer. The upshifting of the $2 \mathrm{D}$ peak after the transfer is a result of the decrease in the stress, as it has been experimentally proved in the recent work of Androulidakis et al. [41]. All the results considering Raman spectra, the $\mathrm{G}$ and $2 \mathrm{D}$ position, the intensity ratio between them, and the $2 \mathrm{D}_{\mathrm{FWHM}}$, are presented in Table 2 in Figure 6. The transfer process should be done carefully to ensure that the graphene film obtained is uniform and without damage. The AFM image (Figure 7) confirms the morphology of the crystal. It has been analyzed with the NOVA software. The software includes tools which allows obtaining the root mean squared roughness of the surface. In more dark color the lobes of the butterfly-like domains are visible, similar to the ones observed by SEM. From the color scale it seems that the graphene domains are dipped inside the surface. We suppose as an explanation that a thin PMMA layer remains on top of the $\mathrm{SiO}_{2}+$ graphene despite the cleaning, affecting the AFM measurement. The PMMA also affects 
TABLE 2: The data of the Raman spectra regarding the $\mathrm{G}$ and $2 \mathrm{D}$ position, the intensity ratio between them, and the $2 \mathrm{D}_{\mathrm{FWHM}}$ are presented.

\begin{tabular}{|c|c|c|c|c|}
\hline Sample & G position $\left(\mathrm{cm}^{-1}\right)$ & 2D position $\left(\mathrm{cm}^{-1}\right)$ & $I_{2 \mathrm{D}} / I_{\mathrm{G}}$ & $2 \mathrm{D}_{\mathrm{FWHM}}\left(\mathrm{cm}^{-1}\right)$ \\
\hline Graphene on copper & 1578 & 2671 & 1,62 & 48 \\
\hline After transfer to $\mathrm{SiO}_{2}$ & 1582 & 2679 & 2,5 & 45 \\
\hline After transfer to quartz & 1587 & 2679 & 1,74 & 48 \\
\hline
\end{tabular}

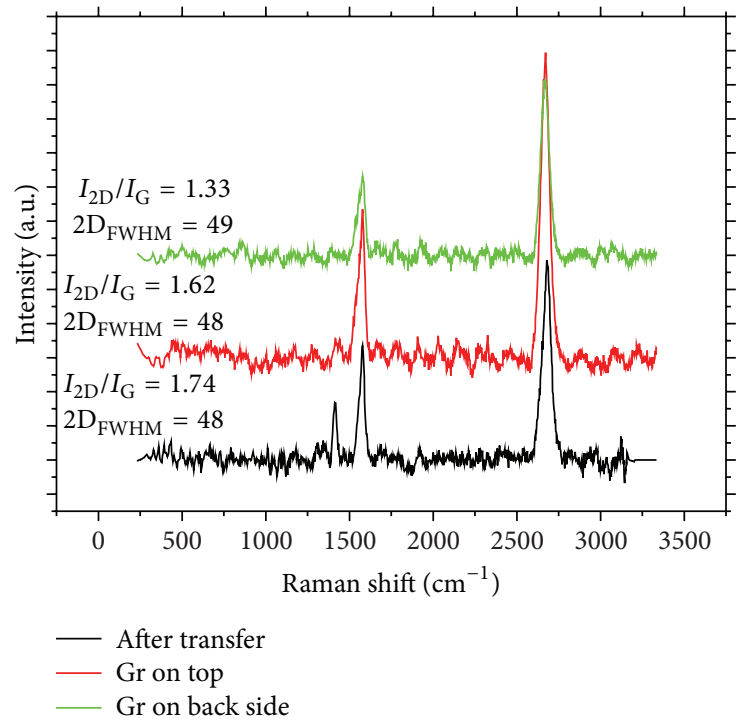

(a)

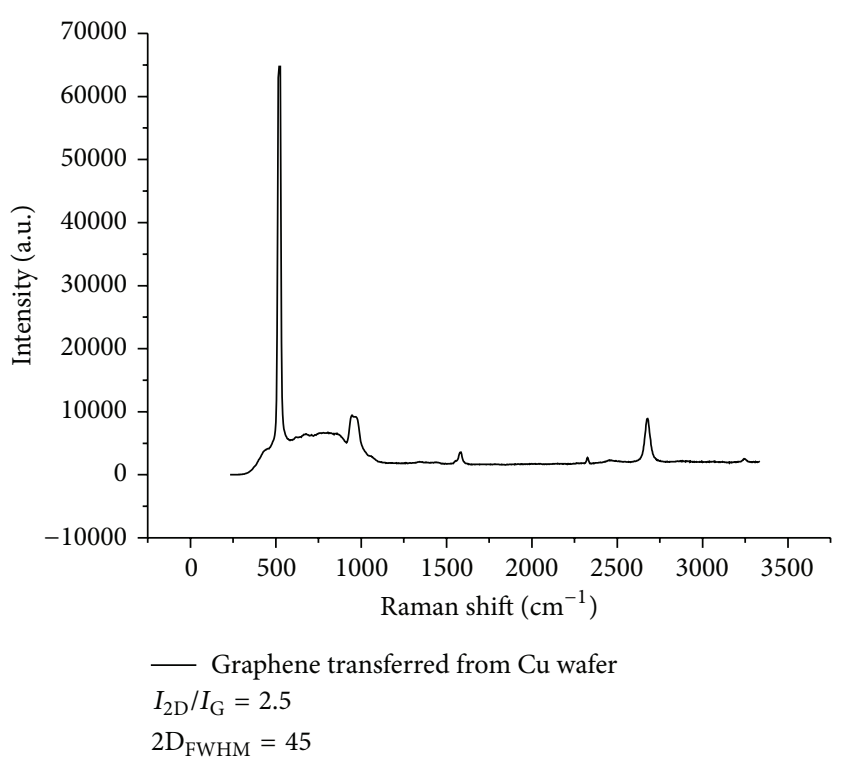

(b)

FIGURE 6: (a) Raman spectra of graphene as growth on copper foil (red line), after transfer to glass (black line) (with thermally grown quartz on top of the glass) and in the back side of the foil (green line). After the transfer a small D peak appears in the spectra, probably a result of defects introduced during the transfer. In the spectra obtained directly in the copper foil the background noise of the copper has been removed. (b) This spectrum corresponds to graphene after the transfer on top of $\mathrm{SiO}_{2} / \mathrm{Si}$. The characteristics $\mathrm{D}+\mathrm{D}^{\prime \prime}$ peak is also visible.

the roughness measurement of the graphene domains. The root mean squared (RMS) roughness of the inside of white box in Figure 7 is $0,78 \mathrm{~nm}$. In other works CVD graphene that presents an RMS roughness of 4,6 nm has been reported (after transfer to $\mathrm{SiO}_{2}$ ). With a careful PMMA cleaning by annealing $t 300^{\circ} \mathrm{C}$ this value could decrease to $0,6 \mathrm{~nm}$ [42]. The value we obtained is close to this value. Some white spots have their origin to etchant or PMMA residuals and despite the careful cleaning are difficult to be completely removed. The homogeneity in the crystal roughness indicates that no second layer of graphene is grown in any part. Some cracks are visible, probably introduced during the transfer process. The image is taken after the transfer of the graphene on top of $\mathrm{SiO}_{2}$. In the graph the profile of a $1 \times 1 \mu \mathrm{m}^{2}$ area is cited in the interior of the graphene domain.

The important role of hydrogen plasma, used for etching the copper foil, was revealed when the growth of graphene was carried out under the same conditions as described for sample A, without any previous ion pretreatment in the copper foil (sample D). This time a precleaning process in a $\mathrm{HNO}_{3}$ /distilled water solution is being performed as described in the work of Kim et al. [43]. Figure 8 shows the SEM pictures of the graphene domains obtained. The nucleation density remains high but the crystals are not well formed as in the graphene obtained in the previous samples.
We considered two possible explanations to this. The first is that, with the chemical etching, the native copper oxide film was not completely removed. The other is that the oxidation of the sample takes place during the cooling of it, which is taking place in high vacuum in absence of hydrogen or any other gas, resulting in the formation of copper oxide islands along with the graphene [44].

\section{Conclusions}

The ability of more sufficient control in the growth of graphene is being explored. The hydrogen flow appears to perform an etching in the graphene domains which affects their morphology and uniformity. The results reveal that a sufficient graphene growth is possible when we optimize the switching of the carbon precursor/hydrogen flow ratio during the process. This reduces the etching effect that the hydrogen is performing, allowing the growth of graphene and the full cover of the substrate by it. Large area graphene is necessary when it comes to large scale applications followed by a careful transfer, to avoid the damaging of the graphene film. The Raman spectroscopy ensures us about the single layer thickness of the graphene film. Performing the Raman measurements on top of different substrates (copper foil, glass, and $\mathrm{SiO}_{2}$ ) does not affect significantly the shape 

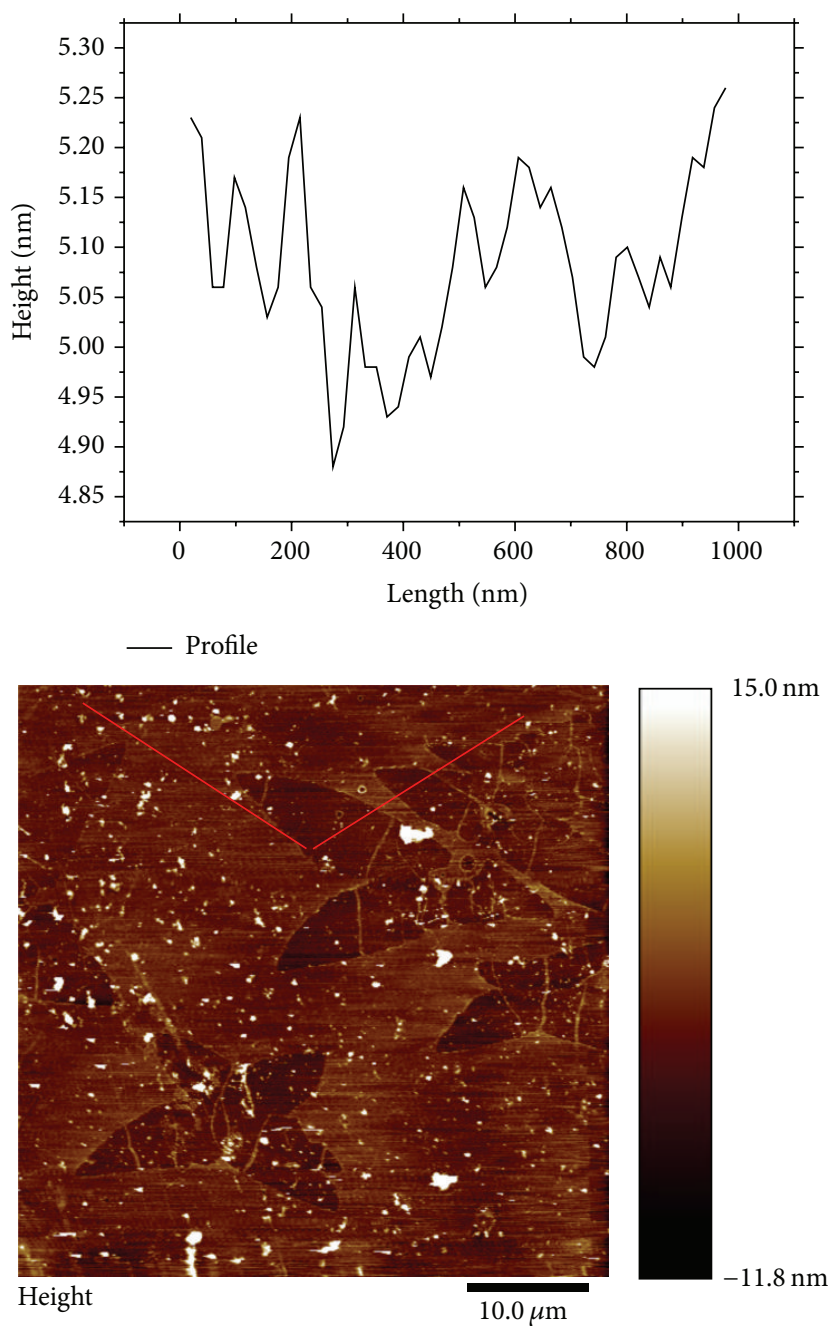

FIGURE 7: AFM image of the graphene transferred on top of $\mathrm{Si} / \mathrm{SiO}_{2}$. The lobes of the crystal can be distinguished. The roughness reveals good homogeneity and absence of second layer growth. Some cracks are introduced by the transfer process. The white spots are impurities introduced by the PMMA, difficult to get completely cleaned. In the up part we see the profile of a $1 \times 1 \mu \mathrm{m}$ area (the indicated square) with RMS roughness $=0,78 \mathrm{~nm}$.

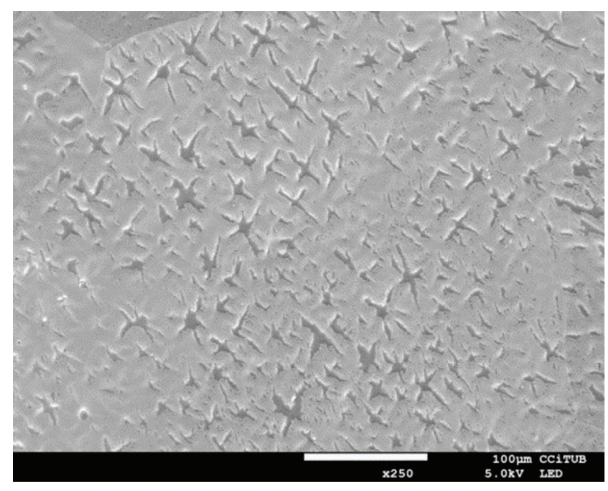

FIGURE 8: Scanning electron microscope picture of graphene grown on copper foil that has not been pretreated with hydrogen plasma etching (sample D). The growth time is 20 minutes. The scale bar has $100 \mu \mathrm{m}$. The copper oxide presence does not allow the formation of large continuous domains. and ratio of the characteristic graphene peaks. In addition the important role of the substrate pretreatment is being investigated. Performing hydrogen plasma before the growth reduces efficiently and faster the native copper oxide layer so that large graphene crystals can be grown.

\section{Conflict of Interests}

The authors declare that there is no conflict of interests regarding the publication of this paper.

\section{Acknowledgments}

The author is supported by the Greek State Scholarships Foundation. The author would like to thank the CCiTUB for the help with the structural and morphological characterization. This work was developed in the frame of the Project 
2014SGR984 of AGAUR from the Generalitat de Catalunya and the Projects MAT2010-20468 and ENE2014-56109-C3-1R of MICINN from Spanish Government.

\section{References}

[1] Y. Zhang, L. Zhang, and C. Zhou, "Review of chemical vapor deposition of graphene and related applications," Accounts of Chemical Research, vol. 46, no. 10, pp. 2329-2339, 2013.

[2] A. K. Geim and K. S. Novoselov, “The rise of graphene," Nature Materials, vol. 6, no. 3, pp. 183-191, 2007.

[3] C. Lee, X. Wei, J. W. Kysar, and J. Hone, "Measurement of the elastic properties and intrinsic strength of monolayer graphene," Science, vol. 321, no. 5887, pp. 385-388, 2008.

[4] J.-H. Chen, C. Jang, S. Xiao, M. Ishigami, and M. S. Fuhrer, "Intrinsic and extrinsic performance limits of graphene devices on $\mathrm{SiO}_{2}$," Nature Nanotechnology, vol. 3, no. 4, pp. 206-209, 2008.

[5] R. R. Nair, P. Blake, A. N. Grigorenko et al., "Fine structure constant defines visual transparency of graphene," Science, vol. 320, no. 5881, p. 1308, 2008.

[6] L. Gomez De Arco, Y. Zhang, C. W. Schlenker, K. Ryu, M. E. Thompson, and C. Zhou, "Continuous, highly flexible, and transparent graphene films by chemical vapor deposition for organic photovoltaics," ACS Nano, vol. 4, no. 5, pp. 2865-2873, 2010.

[7] H. Park, P. R. Brown, V. Bulović, and J. Kong, "Graphene as transparent conducting electrodes in organic photovoltaics: studies in graphene morphology, hole transporting layers, and counter electrodes," Nano Letters, vol. 12, no. 1, pp. 133-140, 2012.

[8] S. Bae, H. Kim, Y. Lee et al., "Roll-to-roll production of 30-inch graphene films for transparent electrodes," Nature Nanotechnology, vol. 5, no. 8, pp. 574-578, 2010.

[9] K. S. Kim, Y. Zhao, H. Jang et al., "Large-scale pattern growth of graphene films for stretchable transparent electrodes," Nature, vol. 457, no. 7230, pp. 706-710, 2009.

[10] F. Schedin, A. K. Geim, S. V. Morozov et al., "Detection of individual gas molecules adsorbed on graphene," Nature Materials, vol. 6, no. 9, pp. 652-655, 2007.

[11] S. Stankovich, D. A. Dikin, R. D. Piner et al., "Synthesis of graphene-based nanosheets via chemical reduction of exfoliated graphite oxide," Carbon, vol. 45, no. 7, pp. 1558-1565, 2007.

[12] C. Berger, Z. Song, X. Li et al., "Electronic confinement and coherence in patterned epitaxial graphene," Science, vol. 312, no. 5777, pp. 1191-1196, 2006.

[13] K. S. Novoselov, A. K. Geim, S. V. Morozov et al., "Electric field effect in atomically thin carbon films," Science, vol. 306, no. 5696, pp. 666-669, 2004.

[14] X. Li, C. W. Magnuson, A. Venugopal et al., "Large-area graphene single crystals grown by low-pressure chemical vapor deposition of methane on copper," The Journal of the American Chemical Society, vol. 133, no. 9, pp. 2816-2819, 2011.

[15] X. Li, W. Cai, J. An et al., "Large-area synthesis of high-quality and uniform graphene films on copper foils," Science, vol. 324, no. 5932, pp. 1312-1314, 2009.

[16] S. Bhaviripudi, X. Jia, M. S. Dresselhaus, and J. Kong, "Role of kinetic factors in chemical vapor deposition synthesis of uniform large area graphene using copper catalyst," Nano Letters, vol. 10, no. 10, pp. 4128-4133, 2010.

[17] X. Li, C. W. Magnuson, A. Venugopal et al., "Graphene films with large domain size by a two-step chemical vapor deposition process," Nano Letters, vol. 10, no. 11, pp. 4328-4334, 2010.

[18] R. Kato, K. Tsugawa, T. Yamada, M. Ishihara, and M. Hasegawa, "Improvement of multilayer graphene synthesis on copper substrate by microwave plasma process using helium at low temperatures," Japanese Journal of Applied Physics, vol. 53, no. 1, Article ID 015505, 2014.

[19] S. Hussain, R. Amade, H. Moreno, and E. Bertran, "RFPECVD growth and nitrogen plasma functionalization of CNTs on copper foil for electrochemical applications," Diamond \& Related Materials, vol. 49, pp. 55-61, 2014.

[20] G. Eres, M. Regmi, C. M. Rouleau et al., "Cooperative island growth of large area single-crystal graphene by chemical vapor deposition on Cu," ACS Nano, vol. 8, no. 6, pp. 5657-5669, 2014.

[21] J.-H. Choi, Z. Li, P. Cui et al., "Drastic reduction in the growth temperature of graphene on copper via enhanced London dispersion force," Scientific Reports, vol. 3, article 1925, 2013.

[22] H. Wang, G. Wang, P. Bao et al., "Controllable synthesis of submillimeter single-crystal monolayer graphene domains on copper foils by suppressing nucleation," Journal of the American Chemical Society, vol. 134, no. 8, pp. 3627-3630, 2012.

[23] A. Michon, S. Vézian, E. Roudon et al., "Effects of pressure, temperature, and hydrogen during graphene growth on $\mathrm{SiC}(0001)$ using propane-hydrogen chemical vapor deposition," Journal of Applied Physics, vol. 113, Article ID 203501, 2013.

[24] J. D. Getty and March Plasma Systems, How Plasma-Enhanced Surface Modification Improves the Production of Microelectronics and Optoelectronics, Chip Scale Review, 2002.

[25] Z. Raskova, F. Krcma, M. Klima, and J. Kousal, "Diagnostic of plasma chemical treatment of archaeological artefacts," Czechoslovak Journal of Physics, vol. 52, pp. D927-D932, 2002.

[26] Y. M. Chang, J. Leu, B. H. Lin, Y. L. Wang, and Y. L. Cheng, "Comparison of $\mathrm{H}_{2}$ and $\mathrm{NH}_{3}$ treatments for copper interconnects," Advances in Materials Science and Engineering, vol. 2013, Article ID 825195, 7 pages, 2013.

[27] S. Chen, L. Brown, M. Levendorf et al., "Oxidation resistance of graphene-coated $\mathrm{Cu}$ and $\mathrm{Cu} / \mathrm{Ni}$ alloy," ACS Nano, vol. 5, no. 2, pp. 1321-1327, 2011.

[28] Y. Wang, Y. Zheng, X. Xu et al., "Electrochemical delamination of CVD-grown graphene film: toward the recyclable use of copper catalyst," ACS Nano, vol. 5, no. 12, pp. 9927-9933, 2011.

[29] I. Vlassiouka, P. Fulviob, H. Meyerc et al., "Large scale atmospheric pressure chemical vapor deposition of graphene," Carbon, vol. 54, pp. 58-67, 2013.

[30] E. Meca, J. Lowengrub, H. Kim, C. Mattevi, and V. B. Shenoy, "Epitaxial graphene growth and shape dynamics on copper: phase-field modeling and experiments," Nano Letters, vol. 13, no. 11, pp. 5692-5697, 2013.

[31] Y. Zhang, Z. Li, P. Kim, L. Zhang, and C. Zhou, "Anisotropic hydrogen etching of chemical vapor deposited graphene," ACS Nano, vol. 6, no. 1, pp. 126-132, 2012.

[32] X. Zhang, J. Ning, X. Li et al., "Hydrogen-induced effects on the CVD growth of high-quality graphene structures," Nanoscale, vol. 5, no. 18, pp. 8363-8366, 2013. 
[33] X. Li, W. Cai, L. Colombo, and R. S. Ruoff, "Evolution of graphene growth on $\mathrm{Ni}$ and $\mathrm{Cu}$ by carbon isotope labeling," Nano Letters, vol. 9, no. 12, pp. 4268-4272, 2009.

[34] S. Kasap, H. Khaksaran, S. Çelik, H. Özkaya, C. Yanık, and I. I. Kaya, "Controlled growth of large area multilayer graphene on copper by chemical vapour deposition," Physical Chemistry Chemical Physics, vol. 17, no. 35, pp. 23081-23087, 2015.

[35] I. Vlassiouk, M. Regmi, P. Fulvio et al., "Role of hydrogen in chemical vapor deposition growth of large single-crystal graphene," ACS Nano, vol. 5, no. 7, pp. 6069-6076, 2011.

[36] Y. Jin, B. Hu, Z. Wei et al., "Roles of $\mathrm{H}_{2}$ in annealing and growth times of graphene CVD synthesis over copper foil," Journal of Materials Chemistry A, vol. 2, no. 38, pp. 16208-16216, 2014.

[37] A. C. Ferrari, J. C. Meyer, V. Scardaci et al., "Raman spectrum of graphene and graphene layers," Physical Review Letters, vol. 97, no. 18, Article ID 187401, 2006.

[38] S. D. Costa, A. Righi, C. Fantini et al., "Resonant Raman spectroscopy of graphene grown on copper substrates," Solid State Communications, vol. 152, no. 15, pp. 1317-1320, 2012.

[39] W. Regan, N. Alem, B. Alemán et al., "A direct transfer of layerarea graphene," Applied Physics Letters, vol. 96, no. 11, Article ID 113102, 2010.

[40] J. Hong, M. K. Park, E. J. Lee, D. Lee, D. S. Hwang, and S. Ryu, "Origin of new broad raman D and G Peaks in annealed graphene," Scientific Reports, vol. 3, article 2700, 2013.

[41] C. Androulidakis, G. Tsoukleri, N. Koutroumanis et al., "Experimentally derived axial stress-strain relations for twodimensional materials such as monolayer graphene," Carbon, vol. 81, no. 1, pp. 322-328, 2015.

[42] A. Pirkle, J. Chan, A. Venugopal et al., "The effect of chemical residues on the physical and electrical properties of chemical vapor deposited graphene transferred to $\mathrm{SiO}_{2}$," Applied Physics Letters, vol. 99, no. 12, Article ID 122108, 2011.

[43] S. M. Kim, A. Hsu, Y.-H. Lee et al., "The effect of copper precleaning on graphene synthesis," Nanotechnology, vol. 24, no. 36, Article ID 365602, 2013.

[44] Y. Qi, J. R. Eskelsen, U. Mazur, and K. W. Hipps, "Fabrication of graphene with $\mathrm{CuO}$ islands by chemical vapor deposition," Langmuir, vol. 28, no. 7, pp. 3489-3493, 2012. 

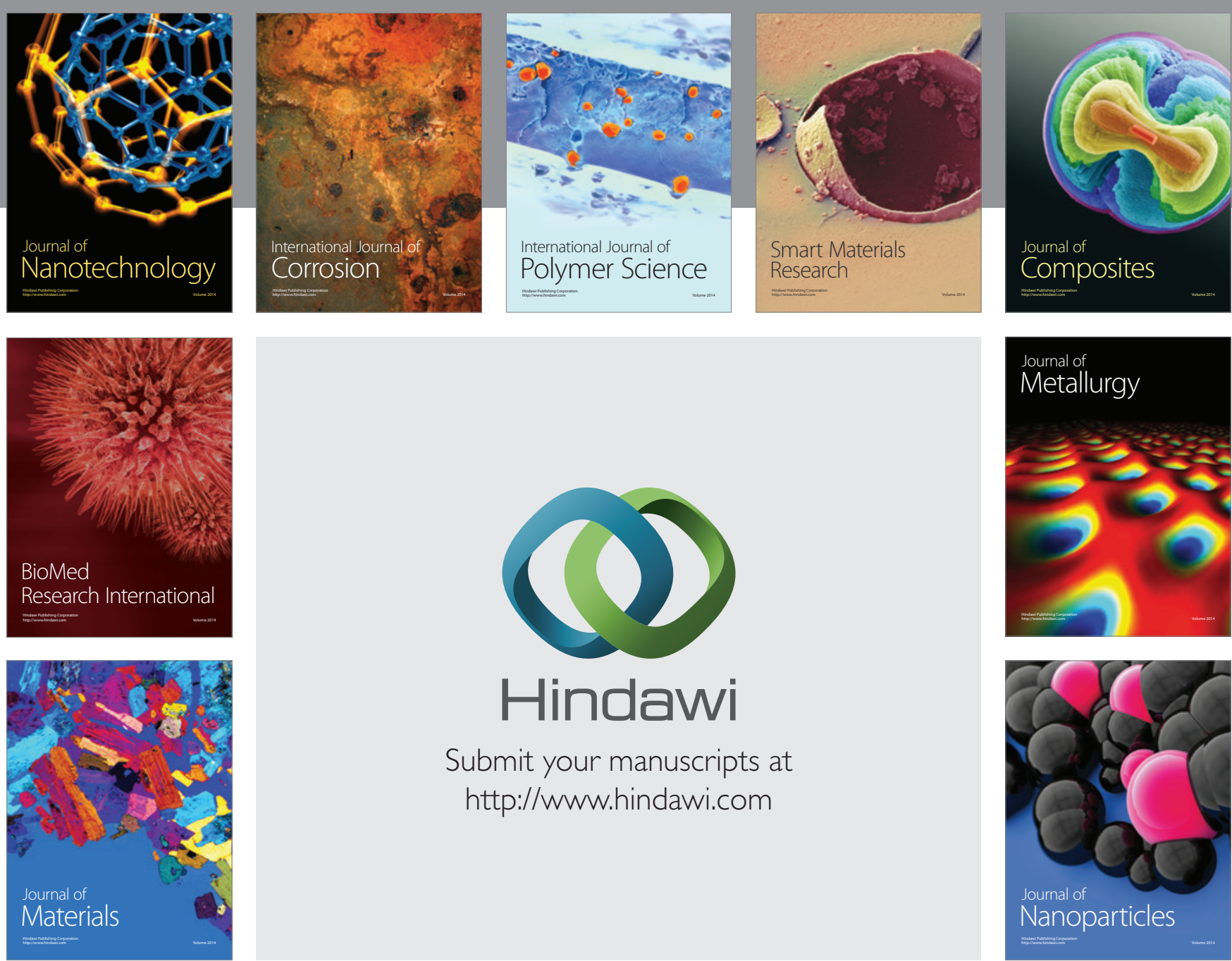

\section{Hindawi}

Submit your manuscripts at

http://www.hindawi.com

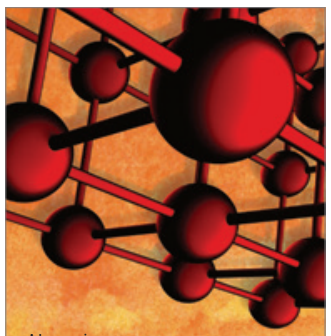

Materials Science and Engineering
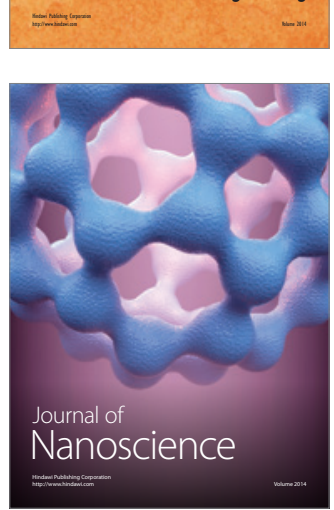
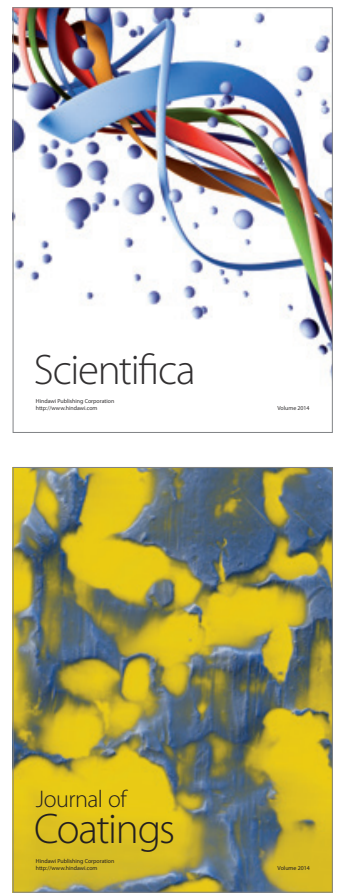
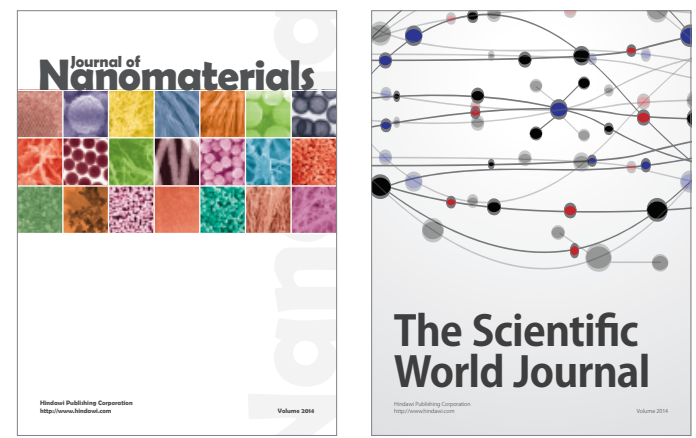

The Scientific World Journal
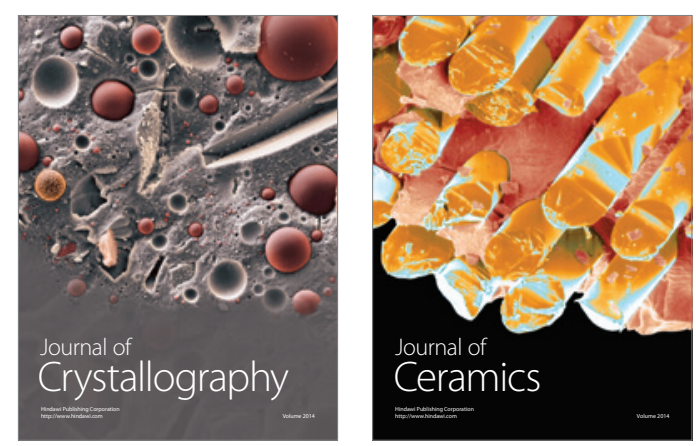
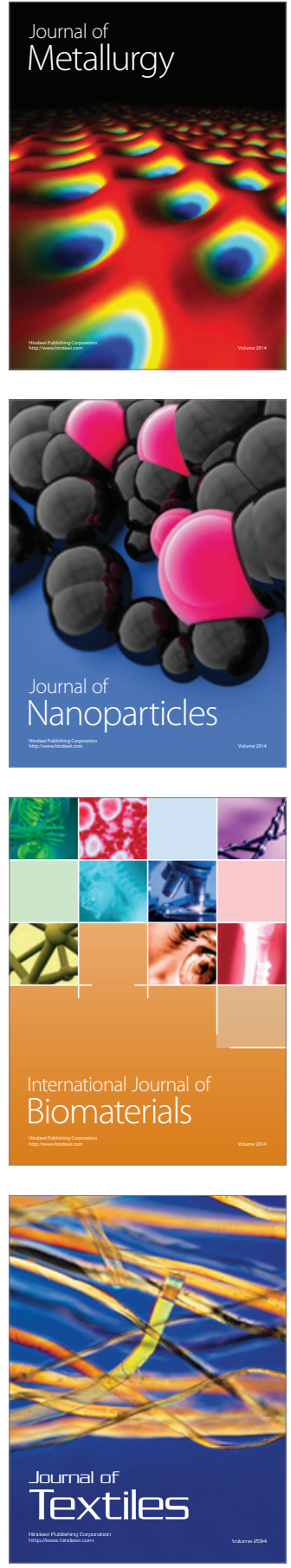